\title{
High-power, Joule-class, temporally shaped multi-pass ring laser amplifier with two Nd:glass laser heads
}

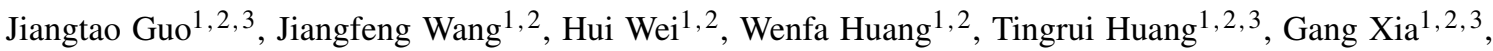 \\ Wei Fan ${ }^{1,2}$, and Zunqi Lin ${ }^{1,2}$ \\ ${ }^{1}$ Key Laboratory of High Power Laser and Physics, Shanghai Institute of Optics and Fine Mechanics, Chinese Academy of Sciences, \\ Shanghai 201800, China \\ ${ }^{2}$ National Laboratory on High Power Laser and Physics, Shanghai Institute of Optics and Fine Mechanics, Chinese Academy of \\ Sciences, Shanghai 201800, China \\ ${ }^{3}$ University of Chinese Academy of Sciences, Beijing 100049, China \\ (Received 26 May 2018; revised 27 October 2018; accepted 30 November 2018)
}

\begin{abstract}
A high-power, Joule-class, nanosecond temporally shaped multi-pass ring laser amplifier system with two neodymiumdoped phosphate glass (Nd:glass) laser heads is demonstrated. The laser amplifier system consists of three parts: an all-fiber structure seeder, a diode-pumped Nd:glass regenerative amplifier and a multi-pass ring amplifier, where the thermally induced depolarization of two laser heads is studied experimentally and theoretically. Following the injection of a square pulse with the pulse energy of $0.9 \mathrm{~mJ}$ and pulse width of $6 \mathrm{~ns}$, a $0.969-\mathrm{J}$ high-energy laser pulse at $1 \mathrm{~Hz}$ was generated, which had the ability to change the waveform arbitrarily, based on the all-fiber structure front end. The experimental results show that the proposed laser system is promising to be adopted in the preamplifier of high-power laser facilities.
\end{abstract}

Keywords: depolarization compensation; laser amplifier; neodymium laser; ring laser

\section{Introduction}

With increasing demand for scientific research and engineering applications in space communication, laser marking and laser material processing, Joule-class laser sources with high gain and energy have been receiving increasing attention. Preamplifiers in high-power laser facilities, such as the National Ignition Facility (NIF), Laser Mega Joule (LMJ), SGII series and the extreme light infrastructure (ELI) ${ }^{[1-3]}$, are representative of these laser systems. As seed sources in the laser inertial confinement fusion (ICF), these preamplifiers are used as boost amplifiers of front-end systems in highpower laser systems, or petawatt pump source systems. In such systems, the gain medium in the main amplifier is always neodymium-doped phosphate glass (Nd:glass), which has excellent optical homogeneity, high doping concentration and flexible geometry ${ }^{[4,5]}$. Thus, Nd:glass remains the best medium for high-energy, high-gain and high extraction efficiency amplifiers.

However, compared with other gain media, the saturation fluence of Nd:glass is relatively higher (saturation fluence

Correspondence to: J. Wang, No. 390 Qinghe Road, Jiading District, Shanghai 201800, China. Email: wajfeng@163.com for Nd:glass: $4.97 \mathrm{~J} / \mathrm{cm}^{2}$; saturation fluence for neodymiumdoped yttrium aluminum garnet (Nd:YAG): $0.66 \mathrm{~J} / \mathrm{cm}^{2}$ ), which indicates a low small-signal gain. The stored energy of Nd:glass is difficult to extract when the injected seed pulses have low energy. In addition, it is inefficient for the structure where injected seed pulses only pass through the gain medium once, just as the traditional traveling wave amplifier does. Thus, the multi-pass amplification structure may be a better alternative for laser amplifiers if the wave-front aberration and parasitic oscillations are well controlled $^{[6,7]}$. Recently, passive multi-pass amplifiers with Faraday rotators (FRs) (or quarter-wave plates (QWPs)) as polarization state controlling elements have been adopted by laboratories ${ }^{[5,8]}$; however, in these amplifiers, the number of passes is limited to four. By accurately designing the optical path, a passive multi-pass active mirror amplifier with more than four passes has been proposed for a lowenergy laser system, such as the booster amplifier in the DiPOLE project ${ }^{[1]}$. In addition, another passive multi-pass slab amplifier based on the angular multiplexing technique was installed in the main amplifier ${ }^{[1,9,10]}$. Nevertheless, the proposed method cannot be applied to rod preamplifiers in high-power laser systems, which require high optical quality, 
flexible control and compactness. Recently, an amplification system that uses Pockels cells (PCs) as active polarization state controlling elements for more than four passes, called active multi-pass amplifier ${ }^{[6,7,11]}$, has been proposed. It mainly comes in two types: linear and ring cavity. Owing to the high energy, high gain and compactness, ring laser amplifiers have been used at high-power laser facilities; an example is the preamplifier in the HALNA100 project, a $0.46 \mathrm{~J}$ system, with a $10 \mathrm{~Hz}$ diode-pumped Nd:YLF ring amplifier that has been developed by using a phase-conjugated wave-front corrector ${ }^{[7]}$. The preamplifier that is used in Omega-EP, CLARA, has two 25.4-mm-diameter Nd:YLF rods, and for each rod, one end is polished to compensate for the transmitted wave-front errors ${ }^{[6,12]}$. However, these amplifiers use Nd:YLF as the gain medium, and Nd:YLF crystals are restricted by the geometry and optical quality of the crystal grown ${ }^{[4]}$. On the other hand, Nd:glass does not have this limitation. Considering the geometries of the gain media and the scalability of laser amplifiers, for ICF applications, Joule-class laser amplifiers based on Nd:glass are more advantageous compared to those based on Nd:YLF.

In this work, we present a multi-pass ring laser system which delivers a $0.969 \mathrm{~J}$ pulse energy, with 6-ns width at $1053 \mathrm{~nm}$, and achieves more than $1000 \times$ amplification with injected pulse energy of $0.9 \mathrm{~mJ}$. The effective extraction efficiency of the stored energy in the gain medium is $33 \%$. The energy stability over $2 \mathrm{~h}$ is $2.4 \%$, while the laser system is operated in the approximately saturated area of the gain medium. The near-field and far-field beam qualities are excellent. The proposed multi-pass ring amplifier is promising to be adopted in preamplifiers of high-power laser facilities in the future.

\section{Description of system setup}

The proposed system is designed for ICF applications, which require high beam quality and flexible beam quality control, including temporal pulse waveforms, pulse spectra, spatial near fields, far fields and wave fronts aberration. The laser system consists of three parts: an all-fiber structure seeder, a regenerative amplifier and a multi-pass amplifier. The entire master oscillator power amplifier (MOPA) system starts from the all-fiber front-end seeder system developed by Qiao et al. ${ }^{[13]}$. The front-end system provides a subnano-Joule energy seed pulse with the central wavelength at $1053 \mathrm{~nm}$ and pulse width of $6 \mathrm{~ns}$. The front-end seeder system can generate arbitrary temporal pulse waveforms with an arbitrary waveform generator (AWG). The generated seed pulse is then transported through the fiber and injected into a highly stable, all-solid-state, diode-pumped Nd:glass regenerative amplifier developed by Wang et al. ${ }^{[14,15]}$. The regenerative amplifier is schematically shown in Figure 1. The amplifier is a linear, folded, 5.4-m long stable cavity, designed to be operated in the stable region. After injection from a fiber collimator (FC), the laser pulse goes through a two-stage isolation, composed of a thin film polarizer

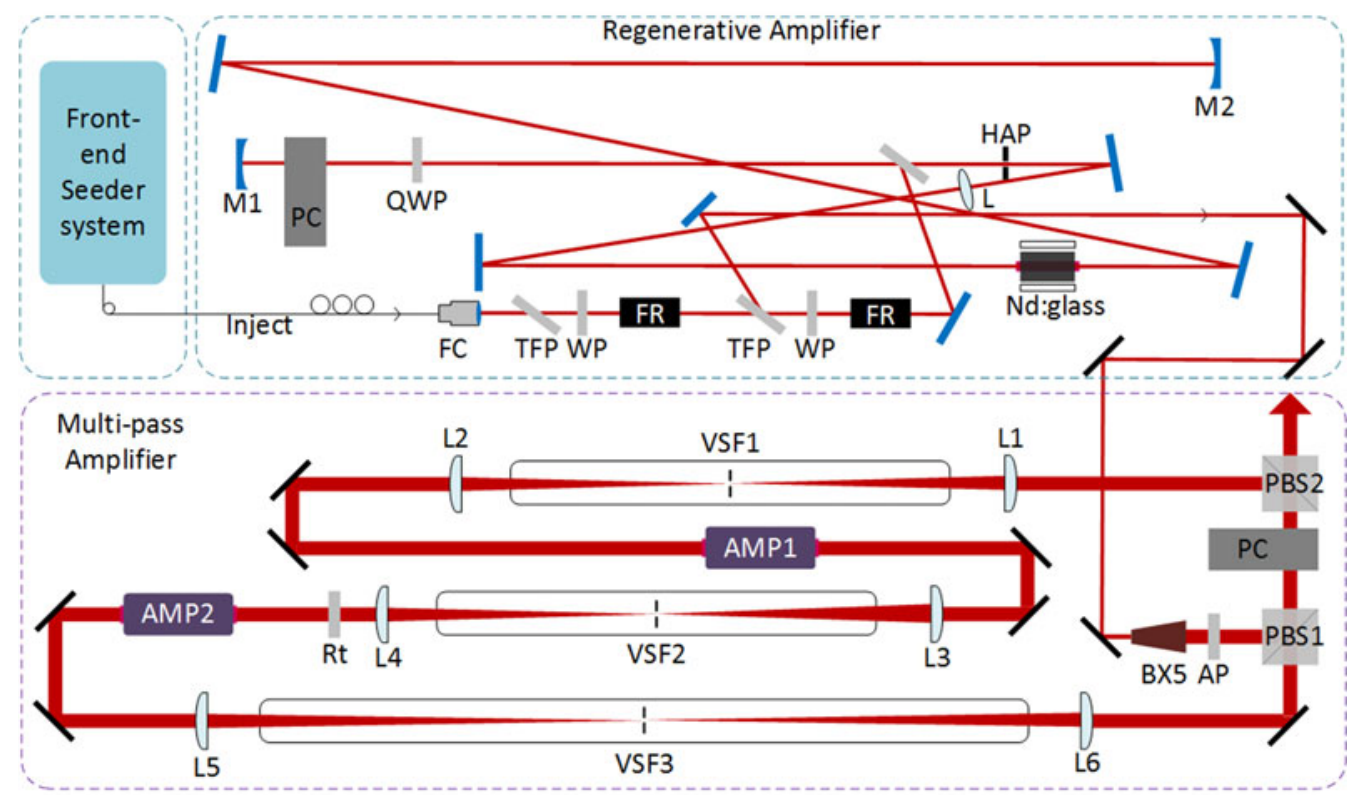

Figure 1. Schematic of the regenerative amplifier and multi-pass amplifier. FC, fiber beam collimator; TFP, thin-film polarizer; WP, half-wave plate; QWP, quarter-wave plate; FR, Faraday rotator; M1, M2, cavity concave mirrors; PC, Pockels cell; AMP, laser head with a diode-pumped Nd:glass rod in the center surrounded by laser diode bars; L, biconvex lens; HAP, hard aperture; BX5, $5 \times$ beam expander; AP, $8.1 \mathrm{~mm} \times 8.1 \mathrm{~mm}$ square soft edge aperture; PBS, polarization beam splitter; L1-L6, plane-convex lenses; VSF1-VSF3, vacuum spatial filters; AMP1, AMP2, two identical laser heads with Nd:glass rod in center surrounded by four xenon lamps. 
(TFP), a half-wave plate (WP) and an FR, to avoid FC damage incurred by the reverse beam (leaking beam from the regenerative cavity, resulting from the low extinction ratio $(200: 1)$ of TFP). The cavity consists of two concave high-reflection end mirrors, M1 and M2, and an intra-cavity convex lens, L. In order to restrict the spatial mode to a single transverse mode, a hard edge aperture (HAP) is set around the intra-cavity lens. The laser pulse round trips in the cavity are controlled by a PC and a QWP, with the PC driver set at a voltage of $1 / 4$ lambda. The regenerative amplifier reaches $10 \mathrm{~mJ}$ class energy, which implies that magnification gain is $10^{7}$. The long-term stability of the output energy is better than $0.5 \%$ r.m.s. The entire system is operated at $1 \mathrm{~Hz}$, owing to the low repetition rate used in high-power laser facilities such as NIF, LMJ and SG series.

Based on the millijoule energy output of the regenerative laser amplifier, the laser pulse is further amplified in a multi-pass ring laser amplifier (MPRA). The structure of the MPRA is outlined in Figure 1. In Figure 1, BX5 indicates a $5 \times$ beam expander. AP is a soft edge aperture with an $8.1 \mathrm{~mm} \times 8.1 \mathrm{~mm}$ square aperture, which is designed to make full use of the 13-mm-diameter gain medium rod. PBS1 and PBS2 are polarization beam splitters combined with the PC, which are used to inject and export the laser pulse, respectively. L1-L4 are 750-mm-focal-length plano-convex lenses, and L5-L6 are 1000-mm-focal-length plano-convex lenses. VSF1-VSF3 are vacuum spatial filters (VSFs) with a $20 \times$ diffraction limitation pinhole in the focal plane of the VSF's first lens, which can filter out high spatial frequency components at the focal plane and suppress ghost images as well as parasitic oscillations resulting from optical elements stray light. The ring cavity was developed based on image relay technique with the three lens pairs and the VSFs. AMP1 and AMP2 are the amplifier laser heads assembled by a $13-\mathrm{mm}$-diameter and 160-mm-long Nd:glass rod at the center surrounded by four xenon flash lamps. The 50mm-diameter $45^{\circ}$ high-reflectivity mirrors with $1053-\mathrm{nm}$ anti-reflection (AR) coatings, are used to fold the cavity. In the MPRA, a PC with deuterated potassium dihydrogen phosphate (DKDP) as the electro-optic crystal (clear aperture $15 \mathrm{~mm}$, model 5046E, FastPulse Technology, Inc.) is used for injecting and switching out the laser pulse. The PC, which is based on the active polarization state control technique, is the key equipment for the active multi-pass amplifier. Rt is a $90^{\circ}$ quartz rotator, used to compensate thermally induced depolarization, combined with a VSF2 $4 \mathrm{f}$ relay-imaged system between two identical laser heads, which will be described in detail in Section 3.

The implementation of the MPRA cavity is briefly discussed below. The entire cavity length $L$ depends on the pulse width $t_{p}$ that is applied to the cavity, $L=t_{c} c>$ $\left(t_{r}+t_{p}\right) c$, where $t_{c}$ is the ring cavity's round trip time, $c$ is the speed of light in air and $t_{r}$ is the rising edge time of the PC (it is assumed that the rising and falling times are $\sim 10 \mathrm{~ns}$ ). In this experiment, the cavity length is approximately $10 \mathrm{~m}$ to support the amplification of a 20-ns maximal laser pulse width, without overlap. The entire system consists of three 4f relay-imaged VSFs. With VSF1, the input image plane (P1), which is the soft edge aperture, is transported to the center of AMP1 (P2). Considering the thermal lens effect, the $\mathrm{P} 2$ position is at the $d_{1}=l / 2 n$ distance from the front end of the rod, where $l$ is the length of the gain medium rod and $n$ is the index of refraction (1.542 for the Nd:glass used in this experiment). In this experiment, the distance from the front end is $5.19 \mathrm{~cm}$. Similarly, P2 has been transported to the center of AMP2 (P3) with VSF2, and P3 has been transported to P4 (between L6 and PBS1) with VSF3. The distances from PBS1 to P4 and PBS1 to AP are the same, which ensures the establishment of the ring system. After transporting $\mathrm{P} 4$, it is regarded as the next initial image plane. Similarly, the image plane will be transported to the output image plane with energy amplified.

\section{Thermal effect compensation of laser heads}

In high-energy, high-power, Joule-class, repetition rate laser amplifier systems, the thermal effect is a major issue that deteriorates the laser beam quality ${ }^{[16,17]}$. Thermal effects, which occur in the gain medium, include thermal lensing and thermal stress-induced birefringence. In our system, to ensure better beam quality output, some methods were adopted to alleviate and compensate for thermal effect, including the design of a high-performance laser head, and compensation for thermal lensing and thermal-induced depolarization.

In our experiment, two identical laser heads were designed. The laser head's cross-section is shown in Figure 2(a). It consists of a gain medium rod, a filter glass sleeve (developed at the Shanghai Institute of Optics and Fine Mechanics (SIOM), Chinese Academy of Sciences), xenon flash lamps, a ceramic diffusive-reflecting cavity, and an outer aluminum alloy. A 13-mm-diameter and 160-mmlength Nd:glass (N3112 developed at SIOM, neodymium concentration $1.2 \%, \mathrm{Nd}_{2} \mathrm{O}_{3}$ by weight) rod at the center is enclosed by a filter glass sleeve pumped by four xenon flash lamps. The deionized water layer between the rod and the filter glass sleeve is $2 \mathrm{~mm}$ thick, for high-speed water flow cooling. To reduce the amount of heat deposited on the gain medium, the filter glass sleeve was used to filter out ultra-violet rays from the xenon flash lamps ${ }^{[5]}$. The ceramic diffusive-reflecting cavity was applied in the pump cavity, which yielded a more uniform pump distribution, compared with a gold-plated cavity or an imaging cavity ${ }^{[16]}$. To obtain a uniform distribution of storage energy in the laser gain medium, parameters determining the characteristics of the pump cavity, such as the rod diameter, lamp diameter and ceramic diffuse reflective geometry, were simulated based on ray tracing method. Finally, considering the manufacturing of ceramics, the design in Figure 2(a) was the one that we used in our experiments. 
(a)

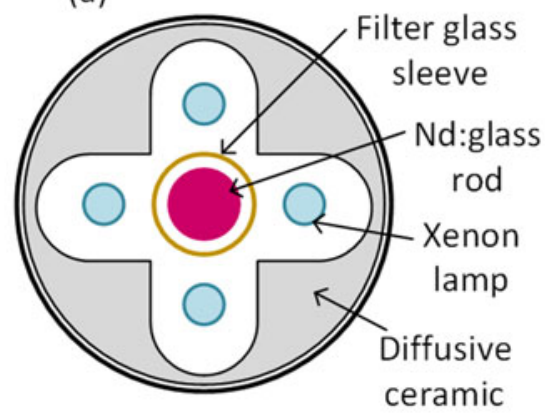

(b)

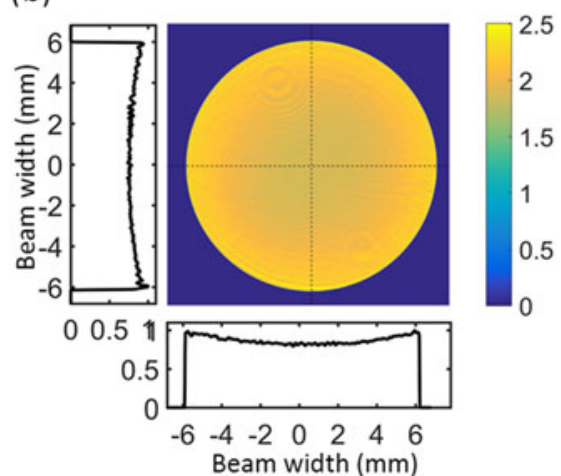

(c)

From Regenerative Amplifier

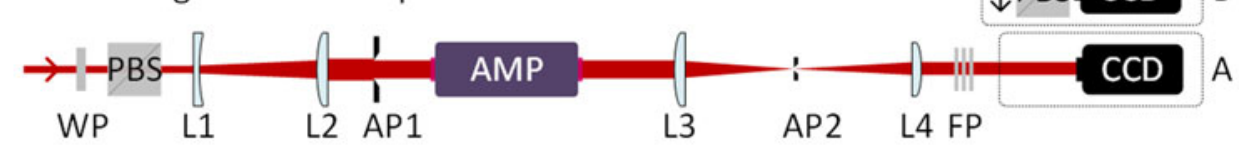

Figure 2. (a) Outline of the laser heads cross-section pumped by four xenon lamps. (b) Two-dimensional small signal gain distribution of the xenon lamp laser head. (c) Outline measurement of the small signal gain and degree of depolarization. WP, half-wave plate; PBS, polarization beam splitter; L1, planoconcave lens with focal length of $-100 \mathrm{~mm}$; L2, plano-convex lens with focal length of $400 \mathrm{~mm}$; AP1, hard edge aperture; AMP, the laser head or some other optical element to be measured; L3, L4, plano-convex lenses with focal lengths of $750 \mathrm{~mm}$ and $500 \mathrm{~mm}$, respectively; AP2, pinhole in the focus plane of L3; FP, neutral density filters and 1053 bandpass filters; A and B are two adjustable options for different measurements, two-dimensional small signal gain distribution and two-dimensional degree of polarization, respectively.

To verify the performance of the two laser heads, the two-dimensional small-signal gain distribution and degree of depolarization (DOP) of the entire cross-section, along with different pump powers, were measured. The measurement setup is shown in Figure 2(c). The laser pulse from the regenerative amplifier passes successively through the WP and the PBS, yielding a line-polarized laser pulse. With the $\mathrm{L} 1(-100 \mathrm{~mm})$ and $\mathrm{L} 2(500 \mathrm{~mm})$, the laser beam is expanded five times. From the hard edge aperture AP1, a beam with an appropriate beam width $(\sim 12 \mathrm{~mm}$ diameter $)$ goes through the laser head. To avoid Fresnel diffraction modulation on the beam periphery, the facet of laser rod is close to the aperture. The beam width is imaged and narrowed to fit the charge-coupled device (CCD) aperture (pixel size $13.3 \mu \mathrm{m} \times 13.3 \mu \mathrm{m}$; number of active pixels $1024 \times 1024$; imaging area $13.6 \mathrm{~mm} \times 13.6 \mathrm{~mm}$ ), with a pair of plane-convex lenses L3 $(750 \mathrm{~mm})$ and L4 $(500 \mathrm{~mm})$, while the beam intensity is controlled by the absorptive neutral density filters FP. For the laser head pumped by the xenon flash lamps, the stray light from the flash lamps and the amplified spontaneous emission (ASE) from the pumped rod severely alter the measurements. Using a pinhole in the focus plane of L3 and bandpass filters in front of the CCD, most of the stray light and ASE can be blocked, ensuring normal and accurate measurements. In Figure 2(c), for twodimensional small-signal gain distribution measurements, the CCD indicated by option A and the method described in Ref. [18] are adopted. For DOP measurements, it is necessary to ensure that the incident beam is vertically polarized, which was obtained by inserting a PBS in front of the laser head. After stabilizing the thermal effects in the laser gain medium (in our setup, usually two minutes after turning on the laser power supply), the measurements started. The two-dimensional DOP $\Gamma_{i, j}$ along the crosssection is defined as follows:

$$
\Gamma_{i, j}=\frac{I_{i, j}(x, y)-I_{i, j \text { out }}(x, y)}{I_{i, j}(x, y)},
$$

where $I_{i, j}(x, y)$ is the two-dimensional output beam intensity distribution, and $I_{i, j \text { out }}(x, y)$ is the two-dimensional output beam intensity distribution after PBS2 is inserted in front of the CCD. The two-dimensional DOP describes point to point beam intensity loss and is useful to investigate which part of the cross-section loses much energy. In practice, it is convenient to consider the total integral $\mathrm{DOP}^{[16]}$,

$$
\gamma=\frac{\sum_{i, j} I_{i, j}(x, y)-I_{i, j \text { out }}(x, y)}{\sum_{i, j} I_{i, j}(x, y)},
$$

which is the ratio of the depolarization beam intensity to the total beam intensity at the output of the gain medium. 
(a)

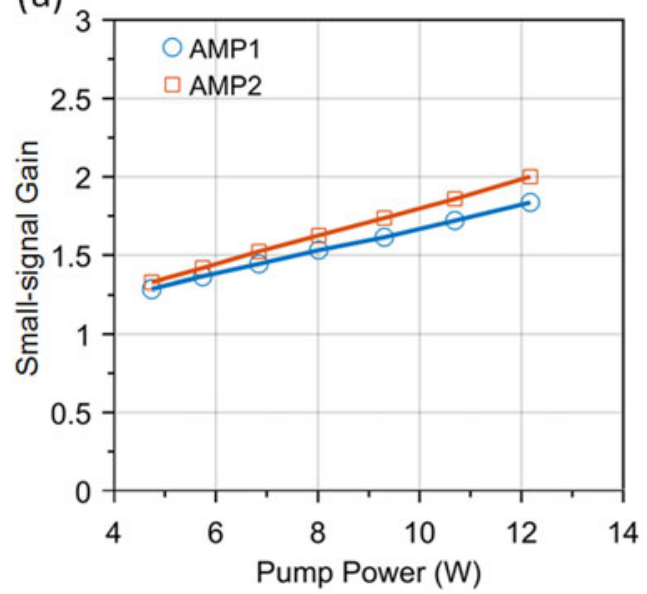

(b)

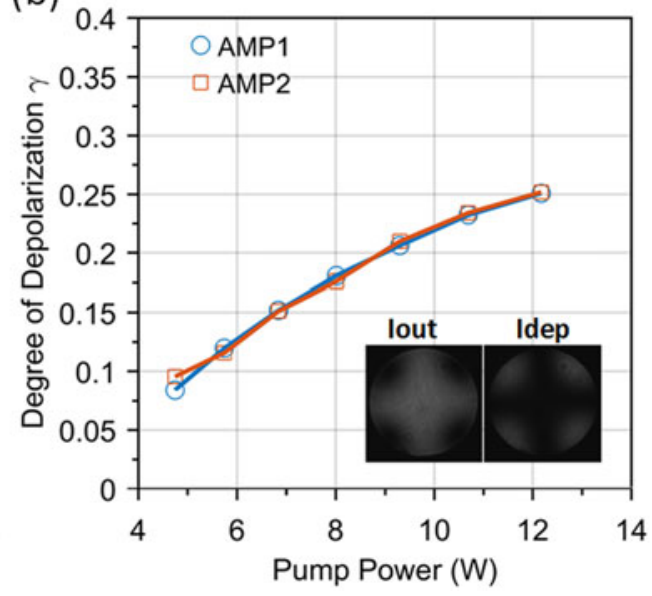

Figure 3. (a) The average small signal gain of the laser rods cross-section versus different pump power and (b) the integral DOP of the laser rods crosssection versus different pump power for the two laser heads, AMP1 and AMP2. The two images (Iout and Idep) in (b) are typical two-dimensional main beam intensity and depolarization beam intensity distribution in measurement.

The results of the two-dimensional small-signal gain distribution for an $800 \mathrm{~V}$ pump of the laser head used in our system is shown in Figure 2(b). The two-dimensional small-signal gain distribution is mostly uniform and the average single-pass small-signal gain is approximately 2.0. The radial direction ratio of the periphery to the center is $1.2: 1$. As expected, the line distribution across the center shows rotational symmetry. The average small-signal gain of the cross-section versus different pump power values is shown in Figure 3(a). The average gain increases almost linearly with increasing pump power. It also indicates that the medium diameter gain medium rod is not limited by the ASE effect, which strongly influences the performance of large aperture Nd:glass disk amplifiers. In addition, the small-signal gain curves of the two laser heads, shown in Figure 3(a), do not overlap completely, while the effective pump power is $12.18 \mathrm{~W}$ (the AMP1 voltage is $715 \mathrm{~V}$; the AMP2 voltage is $800 \mathrm{~V}$ ). This is because the two laser power supply systems are not identical. The electrical capacitances of AMP1 power supply and AMP2 power supply are $500 \mu \mathrm{F}$ and $400 \mu \mathrm{F}$, respectively.

In this experiment, the DOP was measured in detail by using the setup shown in Figure 2(c). The probe beam from the regenerative amplifier passed once through the gain medium, and the beam intensity was recorded with and without PBS. The result is shown in Figure 3(b). The DOPs of the two laser heads are similar, with the same pump power, although they were driven by different laser power supplies. The current pulse duration of the laser power supply at the $10 \%$ point was approximately given by $t_{p}=$ $3 T=3(L C)^{1 / 2}$ (Ref. [16]), and the pulse durations were $396 \mu \mathrm{s}$ and $355 \mu \mathrm{s}$. Thus, the pump efficiency of AMP1 was lower than that of AMP2, though the pump power was the same. In consequence, while the DOPs of the two laser heads are similar (same wasted heat deposited in the laser rods), the small-signal curves do not coincide.

The thermal depolarization induced by the wasted heat deposited in the laser rods significantly deteriorates the beam quality. There are two main conventional types of thermal depolarization compensation methods in high-power laser systems ${ }^{[17,19]}$. Methods in the first category are used in onelaser-head systems that comprise a $45^{\circ} \mathrm{FR}$ or a QWP and a $0^{\circ}$ high-reflectivity mirror; methods in the other category are used in two-identical-laser-head systems with a $90^{\circ}$ quartz rotator combined with an image relay system, which causes the $90^{\circ}$ polarization rotation between the two laser heads. Considering high-power ring cavity laser amplifier systems, the second method for compensating the thermal effect was adopted in this experiment. To understand the process of thermal depolarization compensation, single-pass thermal depolarization after compensation was simulated and measured following the method in Ref. [20]. In Figure 4, the top and bottom panels show the simulated and experimentally measured residual thermal depolarization. In the simulations and experiments $\gamma$ was $0.15 \%$ and $0.30 \%$, respectively, in the area of the injected beam $(\sim 8 \mathrm{~mm} \times 8 \mathrm{~mm})$. Thus, the residual thermal depolarization does not constitute a problem anymore, but it should be kept in mind that the imperfect compensation of depolarization is likely to damage optical elements. In these experiments, we confirm that, to improve the compensation performance, the thermal lens and thermal depolarization should be compensated together ${ }^{[20]}$.

\section{Results and discussion}

The performance of the entire system is illustrated below. When the input energy is $0.9 \mathrm{~mJ}$ and the pulse width is $6 \mathrm{~ns}$, the output energy after eight round trips is approximately 


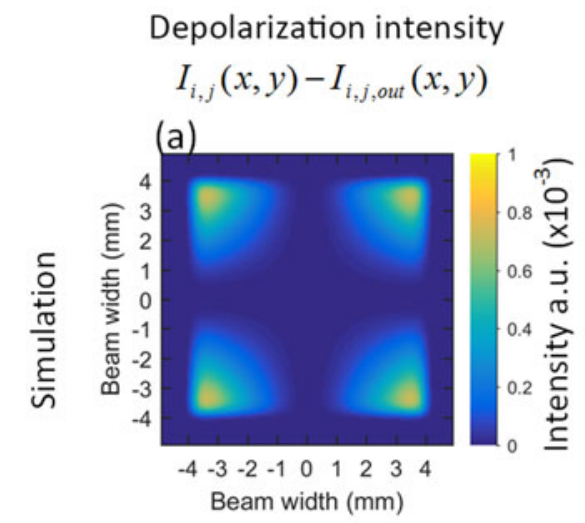

\section{Main beam intensity \\ $I_{i, j, o u t}(x, y)$}

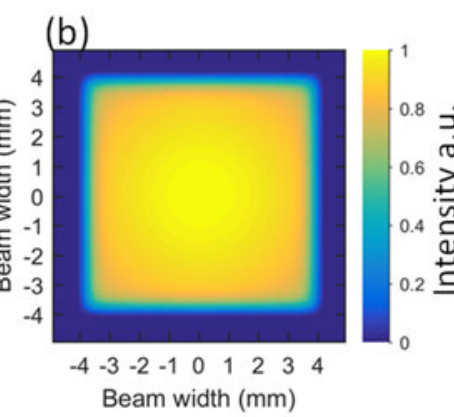

(e)

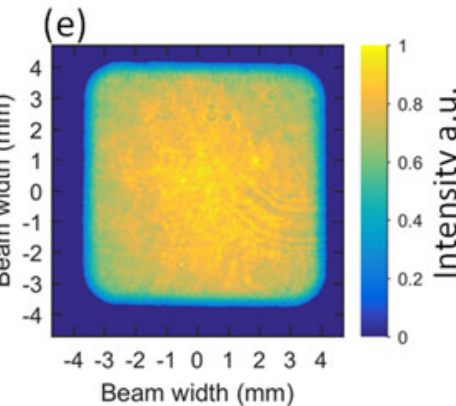

Degree of depolarization

$\Gamma_{i, j}$

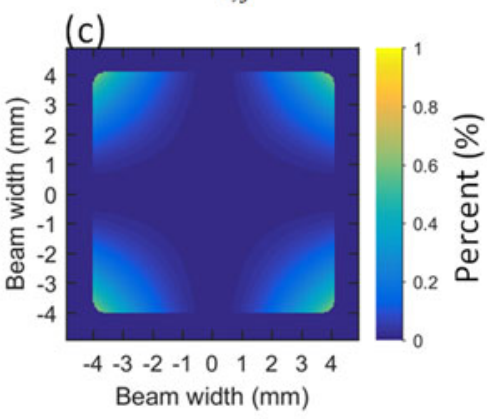

(f)

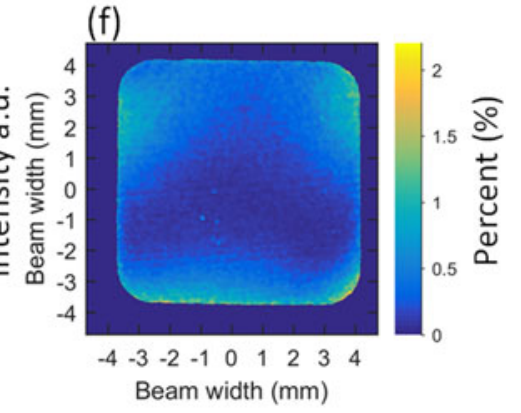

Figure 4. Simulation and experimental measurement of the thermal depolarization compensation. The depolarization output, main beam output and the DOP are shown in (a), (b) and (c), respectively. (d), (e), and (f) show that the experimental measurement produces three types of output.

$0.969 \mathrm{~J}$, which is the target energy of this system. The output fluence density is $1.5 \mathrm{~J} / \mathrm{cm}^{2}$. For safe operation of MPRA, we did not increase the round trips further. The laser cavity has approximately $83 \%$ static single-pass transmittance. The average single-pass effective small-signal gain of the two laser heads is approximately 2.5. The Frantz-Nodvik equation was adopted to simulate and optimize the pulse amplification process ${ }^{[16]}$. The experimental and simulation results for the output energy for the eight round trips are shown in Figure 5, with the blue circles indicating the experimental data, and the red curve indicating the simulation data. Clearly, the maximal energy output increases to $1.5 \mathrm{~J}\left(2.5 \mathrm{~J} / \mathrm{cm}^{2}\right)$ at the 10 th round trip. When the MPRA is operated at the 8th round trip, the energy saturation is approximately attained, which is helpful for improving the system's stability. In the four-energy-level system, the overall stored energy in the laser gain medium is $E_{\mathrm{st}}=g_{0} l E_{s} A=\log G_{0} E_{s} A$, where $g_{0}, G_{0}, E_{s}, l$ and $A$ are, respectively, the small-signal gain coefficient, small-signal gain, saturated energy density, and the length and cross-section of the laser gain medium. The saturated energy density in the laser gain medium is defined as $E_{s}=$ $h \nu / \gamma \sigma=4.967 \mathrm{~J} / \mathrm{cm}^{2}$, where $\gamma=1$ and $\sigma=3.8 \times$ $10^{-20} \mathrm{~cm}^{2}$ for $\mathrm{N} 31 \mathrm{Nd}$ :glass. In our calculations, $G_{0}=3.1$, $l=100 \mathrm{~mm}, A=\pi \times(1.3 / 2)^{2}=1.33 \mathrm{~cm}^{2}$ and the overall stored energy in the laser gain medium is $7.5 \mathrm{~J}$. Thus, the stored energy extraction efficiency $\eta_{\mathrm{ex}}=\left(E_{\mathrm{out}}-E_{\mathrm{in}}\right) / E_{\mathrm{st}} \simeq$ $13 \%$. In addition, we note that the beam overlap efficiency

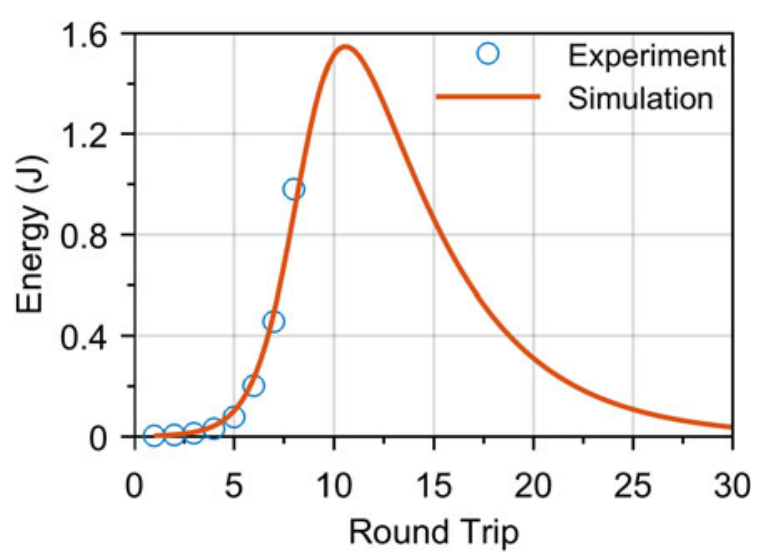

Figure 5. Energy output versus the number of round trips. The blue circles and the red curve correspond to the experimental and simulation results, respectively.

is $\eta_{B}=A_{B} / A=0.8 \times 0.8 \mathrm{~cm}^{2} / 1.33 \mathrm{~cm}^{2}=48.2 \%$ and the single-pass transmittance is $83 \%$. The stored energy of $2.98 \mathrm{~J}$ is extracted by a $0.8 \mathrm{~cm} \times 0.8 \mathrm{~cm}$ square laser beam. Thus, the effective extraction efficiency of the stored energy is approximately $33 \%$.

The near-field and far-field profiles for the MPRA system are shown in Figure 6. The quality of the near field is satisfactory, although no special wave-front correction methods were used, as opposed to the ones in Refs. [7, 12]. Thus, we show that without special wave-front corrections 
(a)

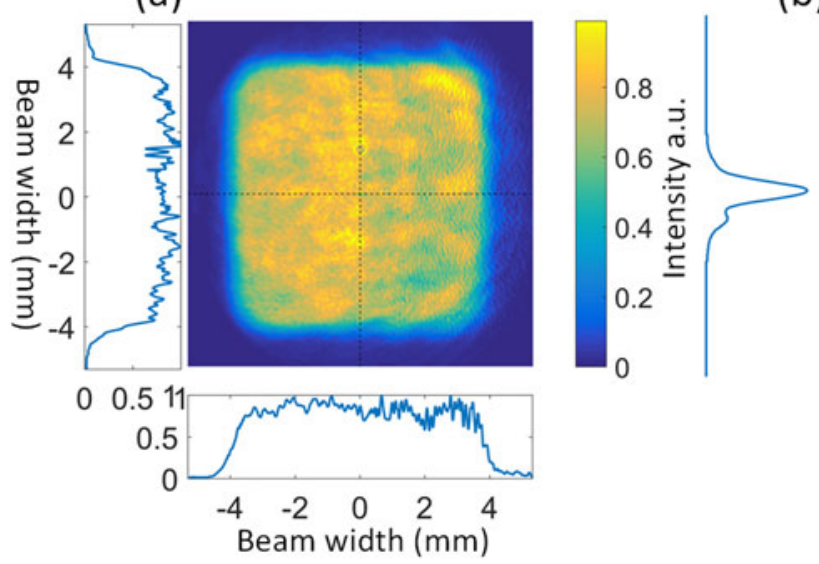

(b)

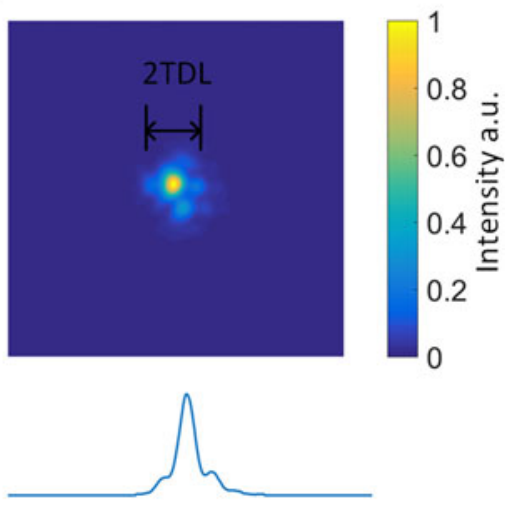

Figure 6. (a) Near field and (b) far field profiles of the multi-pass laser amplifier output at $1 \mathrm{~Hz}, 0.969 \mathrm{~J}$ operation. Also shown are the $x$ and $y$ axis cross-sectional profiles.
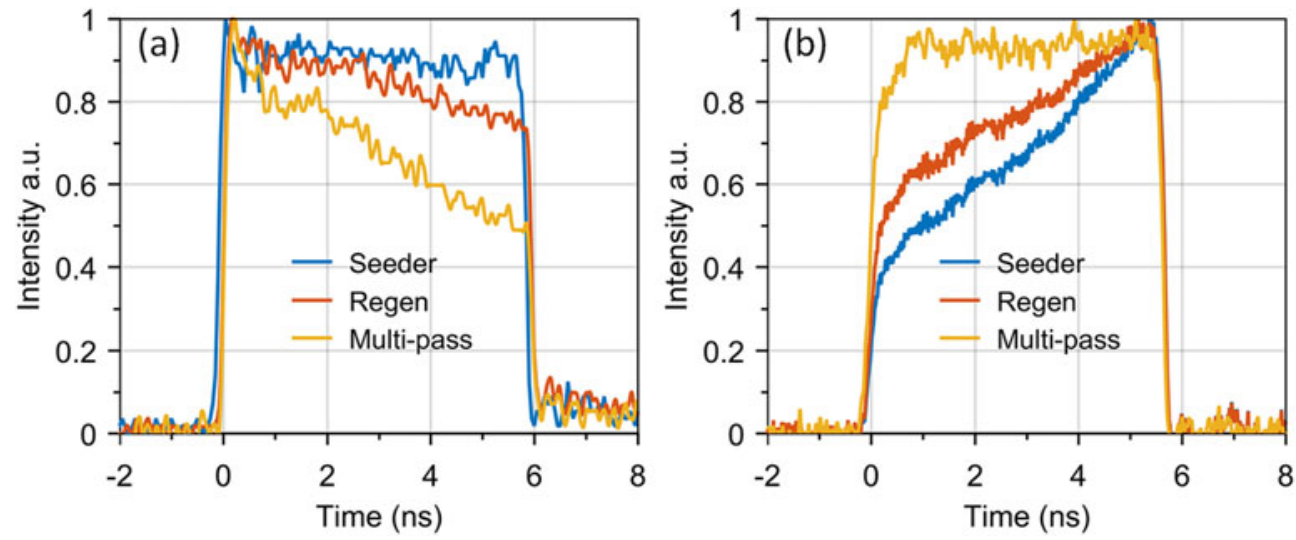

Figure 7. Temporal pulse waveforms of the injection seeder source (Seeder), after passing through the regenerative amplifier (Regen), and after passing through the multi-pass amplifier (Multi-pass), with injected (a) square pulse shape and (b) pre-compensation pulse shape.

satisfactory-quality near field can be obtained by the MPRA system only if the laser head has a perfect pump distribution, which requires an excellent laser head design. The near-field intensity modulation (FM), defined as the ratio of maximal $\left(I_{\max }\right)$ to average $\left(I_{\mathrm{avg}}\right)$ optical intensities inside the beam aperture $\mathrm{FM}=I_{\max } / I_{\mathrm{avg}}$, after eight round trips, is 1.42 . The line distributions in the $x$ and $y$ directions at the center point are also shown in Figure 6(a), which can be fitted by an eighth-order super-Gaussian distribution. The far-field results for the system are shown in Figure 6(b). At the output of the laser system, a thin lens with 1-m focal length was adopted for the far-field measurements. The far-field beam width is $0.133 \mathrm{~mm}$ (full width at half maximum), and $90 \%$ of the far-field energy is concentrated within the two times diffraction limit (TDL).

The temporal pulse waveforms of the entire system are shown in Figure 7; these waveforms were measured with a Tektronix DPO7064B 6G oscilloscope and a Thorlabs DET08CFC high-speed fiber photoelectric detector. When square pulse waveform is injected, the square pulse distortions (SPDs) shown in Figure 7(a), after the two-stage amplifier, were 1.27 and 1.9 , respectively. Owing to the arbitrary shaping ability of the front-end seeder system, the output pulse SPD can be compensated by actively adjusting the injected pulse waveform. A satisfactory square temporal pulse waveform in our multi-pass system can then be obtained, as is shown in Figure 7(b).

\section{Conclusion and outlook}

In conclusion, with the multi-pass ring laser system, $0.969 \mathrm{~J}$ laser pulse energy (pulse width: $6 \mathrm{~ns}$; center wavelength: $1053 \mathrm{~nm}$ ) and $1000 \times$ amplification were achieved for $0.9 \mathrm{~mJ}$ injected pulse energy. The effective extraction efficiency of the stored energy in the laser gain medium was $33 \%$. The energy stability during $2 \mathrm{~h}$ was $2.5 \%$, while the laser system was approximately operated in the saturated regime of the gain medium. The near-field amplitude modulation was 1.4, and $90 \%$ of the far-field energy was focused within 2TDL. Finally, we demonstrated that the multi-pass ring amplifier is promising and can be used in preamplifier systems at largescale laser facilities such as NIF, LMJ and SG II, owing to its high gain, compactness and high extraction efficiency. In 
addition, owing to the low single-pass small-signal gain of laser diode (LD) heads and high cost of LDs, this structure is more appropriate for the diode-pumped solid-state laser system.

In future experiments, laser heads pumped by LD modules will be employed to demonstrate an MPRA system, which enables us to scale the system to develop high-energy and high-power systems for application in preamplifiers of largescale laser facilities or pump source systems for petawattscale systems.

\section{Acknowledgements}

The authors gratefully acknowledge the support of the Shanghai Science and Technology Foundation for Young Scholars (No. 17YF1429600) and the National Natural Science Foundation of China (No. 61705242).

\section{References}

1. S. Banerjee, K. Ertel, P. D. Mason, P. Jonathan Phillips, M. De Vido, J. M. Smith, T. J. Butcher, C. Hernandez-Gomez, R. Justin, S. Greenhalgh, and J. L. Collier, Opt. Express 23, 19542 (2015).

2. C. Danson, D. Hillier, N. Hopps, and D. Neely, High Power Laser Sci. Eng. 3, e3 (2015).

3. P. D. Mason, S. Banerjee, K. Ertel, P. J. Phillips, T. J. Butcher, J. M. Smith, M. De Vido, S. Tomlinson, O. Chekhlov, W. Shaikh, S. Blake, P. Holligan, M. Divoky, J. Pilar, C. Hernandez-Gomez, R. J. S. Greenhalgh, and J. L. Collier, Proc. SPIE 9513, 951302 (2015).

4. L. Hu, S. Chen, J. Tang, B. Wang, T. Meng, W. Chen, L. Wen, J. Hu, S. Li, and Y. Xu, High Power Laser Sci. Eng. 2, e1 (2014).
5. C. Wang, H. Wei, J. Wang, D. Huang, W. Fan, and X. Li, Chin. Opt. Lett. 15, 011401 (2017).

6. V. Bagnoud, M. J. Guardalben, J. Puth, J. D. Zuegel, T. Mooney, and P. Dumas, Appl. Opt. 44, 282 (2005).

7. T. Sekine, S.-i. Matsuoka, R. Yasuhara, T. Kurita, R. Katai, T. Kawashima, H. Kan, J. Kawanaka, K. Tsubakimoto, and T. Norimatsu, Opt. Express 18, 13927 (2010).

8. M. Bowers, S. Burkhart, S. Cohen, G. Erbert, J. Heebner, M. Hermann, and D. Jedlovec, Proc. SPIE 6451, 64511M (2007).

9. M. Divoky, M. Smrz, M. Chyla, P. Sikocinski, P. Severova, O. Novak, J. Huynh, S. S. Nagisetty, T. Miura, J. Pilar, O. Slezak, M. Sawicka, V. Jambunathan, J. Vanda, A. Endo, A. Lucianetti, D. Rostohar, P. D. Mason, P. J. Phillips, K. Ertel, S. Banerjee, C. Hernandez-Gomez, J. L. Collier, and T. Mocek, High Power Laser Sci. Eng. 2, e3 (2014).

10. S. Tokita, M. Divoky, H. Furuse, K. Matsumoto, Y. Nakamura, M. Yoshida, T. Kawashima, and J. Kawanaka, Opt. Mater. Express 4, 2122 (2014).

11. X. Qiao, D. Wang, X. Zhang, F. Zeng, S. Gao, K. Yao, Y. Yang, X. Zhang, Q. Yuan, and J. Zhao, Opt. Express 25, 9848 (2017).

12. V. Bagnoud, J. Puth, J. D. Zuegel, T. Mooney, and P. Dumas, in Advanced Solid-State Photonics (Optical Society of America, 2004), paper MB17.

13. Z. Qiao, X. Wang, W. Fan, and Z. Lin, Opt. Lett. 40, 4214 (2015).

14. J. Wang, X. Li, H. Wei, and J. Zhu, Chin. Phys. Lett. 25, 524 (2008).

15. Y. Peng, J. Wang, Z. Zhang, W. Fan, and X. Li, Chin. Opt. Lett. 12, 041402 (2014).

16. W. Koechner, Solid-State Laser Engineering (Springer, New York, 1999).

17. Q. Lü, N. Kugler, H. Weber, S. Dong, N. Müller, and U. Wittrock, Opt. Quantum Electron. 28, 57 (1996).

18. A. A. Shaykin, A. P. Fokin, A. A. Soloviev, A. A. Kuzmin, I. A. Shaikin, K. F. Burdonov, A. V. Charukhchev, and E. A. Khazanov, Quantum Electron. 44, 426 (2014).

19. R. Fluck, M. R. Hermann, and L. A. Hackel, Appl. Phys. Lett. 76, 1513 (2000).

20. J. Jeong, S. Cho, T. Kim, and T. J. Yu, J. Korean Phys. Soc. 68, 653 (2016). 